\title{
SMA Thin Strip for Rotary-Driving Element
}

\author{
H. Tobushi ${ }^{1, a}$, E. A. Pieczyska, ${ }^{2, b}$, W. K. Nowacki ${ }^{2, c}$ and Y. Sugimoto ${ }^{1, d}$ \\ ${ }^{1}$ Department of Mechanical Engineering, Aichi Institute of Technology, \\ 1247, Yachigusa, Yakusa-cho, Toyota, 470-0392, Japan \\ ${ }^{2}$ Institute of Fundamental Technological Research, Polish Academy of Sciences,
}

21 Swietokrzyska, Warsaw, 00-049, Poland

a tobushi@aitech.ac.jp, ${ }^{b}$ epiecz@ippt.gov.pl, ${ }^{c}$ wnowacki@ippt.gov.pl, ${ }^{\mathrm{d}}$ p07711pp@aitech.ac.jp

Keywords: Shape memory alloy, Thin strip, Torsion, Rotary driving element, Strain energy

\begin{abstract}
If a shape-memory alloy (SMA) thin strip is applied as an element subjected to torsion, a rotary driving element with a simple mechanism can be developed. The torsion tests were carried out for the SMA thin strip. Torque and recovery torque, both increase in proportion to the angle of twist and temperature. The recoverable strain energy increases in proportion to temperature. The dissipated work decreases slightly with an increase in temperature. A means of opening and closing a door with an element driven by an SMA thin strip is demonstrated.
\end{abstract}

\section{Introduction}

In recent years, the development and applications of smart materials have attracted worldwide attention. Shape-memory alloys (SMAs) have played a leading part in research of smart materials ${ }^{[1,}$ ${ }^{2]}$. The main properties of SMAs are the shape memory effect (SME) and superelasticity (SE). Thanks to these SMAs can be used in the driving elements of actuators, robots and heat engines. The SME and SE appear as a result of the martensitic transformation (MT).

The deformation properties of the SME and SE strongly depend on temperature and stress. Because of the adaptable thermal response of SMA elements, thin wires and thin tapes are widely used in practical applications ${ }^{[3]}$. In order to improve the flight performance of the rotor aircraft, twist of the blades was investigated recently by using the torsional deformation of a TiNi SMA tube [4]

In practical applications making use of SMA thin strips, torsional deformation can be obtained simply by holding both ends in the plates without any gripping device. If the characteristics of SE are exploited, a high performance of energy storage can be achieved similar to that of a torsion bar. In this way, simple and small actuators can be developed. In the case of the SMA thin strip twisted by fixing both ends, not only torsional but also tensile stress can be induced along both edges of the strip.

In the present paper, the torsional deformation properties of a TiNi SMA thin strip are investigated in a torsion test with loading and unloading at various temperatures. A means of opening and closing a door driven by an SMA thin strip is demonstrated.

\section{Experimental Method}

Materials and specimen. The materials used in the experiments were Ti-50.18 at $\%$ Ni SMA thin strips (NT-M2) with a thickness of $t=0.25 \mathrm{~mm}$ and a width of $w=5 \mathrm{~mm}$ produced by Furukawa Electric Co., Ltd. The specimen was a uniform flat tape of length $L=60 \mathrm{~mm}$. The gauge length of the specimen was $l=40 \mathrm{~mm}$ and the length held in the gripping device was $10 \mathrm{~mm}$ at each site.

The as-received material was first hot-rolled from a wire with a diameter of $d=3.5 \mathrm{~mm}$ heated at $1073 \mathrm{~K}$ into a strip with a thickness $t=1.5 \mathrm{~mm}$, followed by cold-stripping in several steps into the strip with a thickness of $t=0.25 \mathrm{~mm}$. The hardening mechanism was supplied not by precipitation hardening but by work-hardening. A fat plane was shape-memorized by fixing in a flat plane for 1 min at $803 \mathrm{~K}$ in the furnace. 
The as-received material was heat-treated again in an electric furnace to memorize the flat plane. In this heat-treatment, the as-received material was first fixed in a flat plane, heated up to $673 \mathrm{~K}$ for 2 hours and kept at $673 \mathrm{~K}$ for 1 hour, followed by cooling in the furnace. The same tests were carried out for both the as-received material and the heat-treated material used in the originally undeformed state.

Experimental procedure. The torsion and the recovery torque tests were carried out as follows.

Torsion test. The specimen was fixed onto the grips of the twisting shaft. The torque gauge was fixed on the twisting shaft and the specimen was twisted at the prescribed angle of twist per unit length $\theta$ (total angle of twist $\phi$ ). The angle of twist and torque were measured simultaneously. In the torsion test, the maximum angle of twist per unit length was applied and thereafter torque was completely removed during unloading. The test temperatures $T$ were room temperature (RT), 333, 343 and $353 \mathrm{~K}$. The test was carried out in air at RT and in water at 333, 343 and $353 \mathrm{~K}$. The temperature rise of the specimen during twisting in air was smaller than $4 \mathrm{~K}$. Therefore, the influence of the temperature rise is negligible. The maximum angle of twist per unit length $\theta_{\max }$ was $78.5 \mathrm{rad} / \mathrm{m}$ (maximum total angle of twist $\phi_{\max }=\pi$ ).

Recovery torque test. The specimen was first twisted at room temperature in air up to the prescribed angle of twist per unit length $\theta$ (total angle of twist $\phi$ ). Torque was measured at this angle and the angle of twist was held constant. Maintaining this constant angle of twist, the specimen was soaked in water at the prescribed temperature, and torque was measured in the heated state. The recovery torque used under thermal cycling was determined by the difference of torque between the experimental and room temperatures. The temperature at which the specimen was twisted was room temperature, and the experimental temperatures $T$ were 333,343 and $353 \mathrm{~K}$.

\section{Experimental Results and Discussion}

Deformed state in torsion. In the present study, the strip was twisted from one end while both ends were held constant at the axial position. Photographs of the deformed SMA thin strip are shown in Fig. 1 for each angle of twist. The left side in each case shows the fixed end and the right side shows the twisting end.

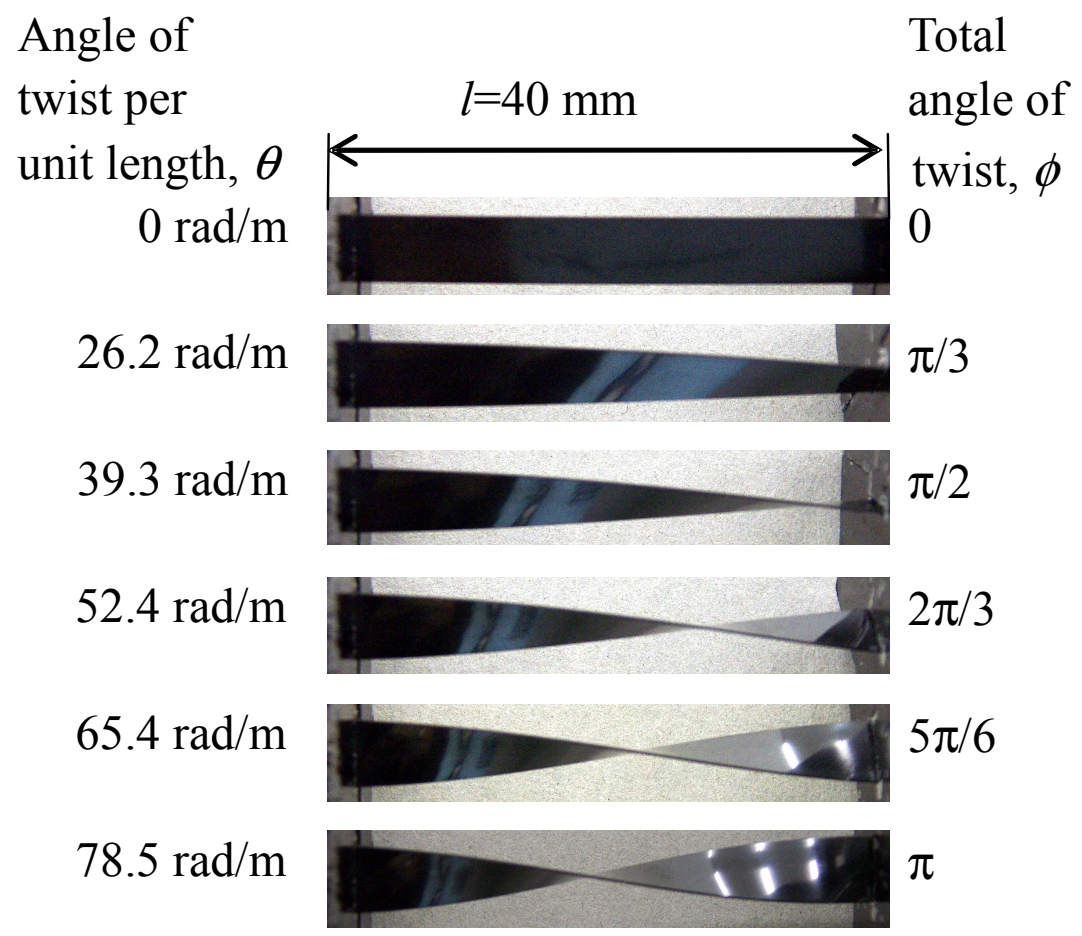

Fig. 1 Photograph of twisted SMA thin strip at each angle of twist. 
As it can be seen, the upper and lower surfaces of the SMA thin strip are reversed during twisting and a crossover point appears in the twisting end at the angle of twist per unit length $\theta=39.3 \mathrm{rad} / \mathrm{m}$ (total angle of twist $\phi=\pi / 2$ ). The crossover point propagates from the twisting end and reaches the central part of the specimen at the angle of twist per unit length with $\theta=78.5 \mathrm{rad} / \mathrm{m}$. Comparing the deformed states at $\theta=0$ and $78.5 \mathrm{rad} / \mathrm{m}(\phi=0$ and $\pi)$, both edges of the thin strip are found to be elongated by twisting since both ends are axially fixed. Therefore, tensile stress is induced along both edges and the stress state becomes much more complex than torsion in a cylindrical bar.

Relationship between torque and angle of twist. The relationship between torque $M$ and angle of twist $\theta$ for the heat-treated material as obtained from the torsion test is shown in Fig. 2.

As it can be seen (Fig. 2a), $M$ increases with an increase in $\theta$. At room temperature, a large residual angle of twist per unit length appears after unloading, proving the SME. At $T=333 \mathrm{~K}$, since $T$ is $A_{s}<T<A_{f}$ and therefore there is a partial effect of superelasticity in which the reverse transformation is not completed. Residual angle of twist appears after unloading. At temperatures above $343 \mathrm{~K}$, the angle of twist recovers during unloading and no residual angle of twist was noticed.

As can be seen from the relationship between torque and temperature in the loading process, shown in Fig. 2(b), torque $M$ increases in proportion to temperature rise $\Delta T$ for the same angle of twist per unit length $\theta$ at temperature range above $A_{s}$. The slope increases in proportion to $\theta$. This relationship is expressed as follows

$$
M=\alpha_{1} \theta\left(T-M_{s}\right),
$$

where $M_{s}$ denotes the MT starting temperature without stress and $M_{s}=305 \mathrm{~K}$. This relationship appears owing to the fact that the MT stress increases proportionally to temperature $\left(T-M_{s}\right)$.

In the case of torsion in a bar of round cross-section, with diameter $d$, the angle of twist $\phi$ is given by the equation, $\phi=M l / G I_{p}$ where $G$ and $I_{p}=\pi d^{4} / 32$ are the modulus of rigidity and the polar area moment of inertia, respectively. For a bar of rectangular cross-section (width $w$ and thickness $t$ ), the angle of twist per unit length $\theta$ is expressed as follows ${ }^{[5]}$

$$
\theta=\frac{\phi}{l}=\frac{M}{k_{1} w t^{3} G},
$$

where the coefficient $k_{1}$ depends on the $w / t$ ratio. Considering the fact that the $M-\theta$ curves during loading shown in Fig. 2(a) are close to straight lines, Eq. (1) becomes

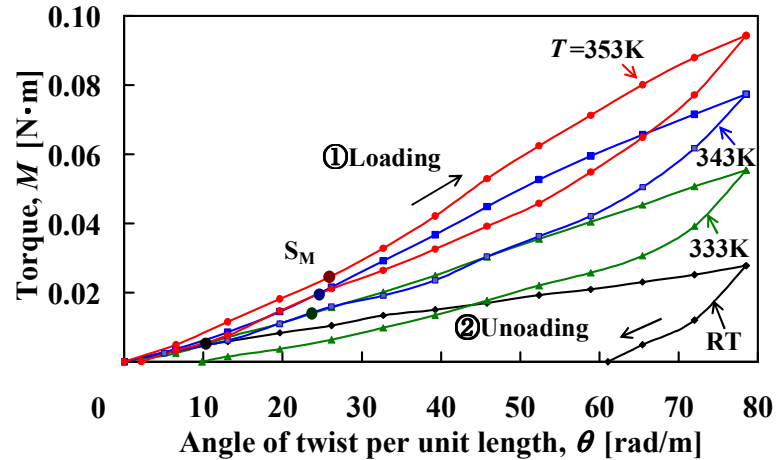

(a) Relation between torque and angle of twist.

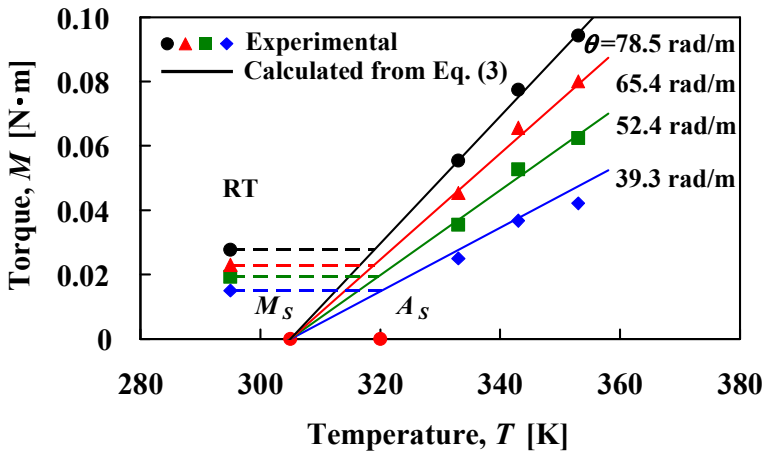

(b) Relation between torque and temperature in the loading process.

Fig. 2 Results of torsion test for heat-treated material 


$$
M=a w t^{3} G \theta\left(T-M_{s}\right),
$$

where the factor $a$ depends on the ratio $w / t$. The values of $G$ at temperatures above and below $A_{s}$ differ in a ratio of about $3: 1$. Taking the average value $G=20 \mathrm{GPa}$ and $a=1.61 \times 10^{-2} \mathrm{~K}^{-1}$, the calculated results can be found as shown by the solid lines in Fig. 2(b). As can be seen, the dependence of $M$ on $\theta$ and $T$ is well described by Eq. (3). As expressed in Eq. (3), the effect of thickness $t$ on $M$ is significantly greater than the influence of $w$.

Estimation of MT starting point. The starting point of the MT can be estimated based on the Mises-yield theory for the MT stress which has been validated for a TiNi SMA from experiments done on a tube under complex loading ${ }^{[6]}$. The torsional transformation stress $\tau_{M}$ is determined from the MT starting stress $\sigma_{M}$ obtained from the tension test, as $\tau_{M}=\sigma_{M} / \sqrt{3}$. The torque at the start of the MT is calculated using the torsional stress obtained from the theory of elasticity for a thin strip $^{[5]}$. The relationship between torque $M$ and the maximum torsional stress $\tau_{\max }$ in the elastic region of the rectangular-section bar can be expressed as follows

$$
M=k_{2} w t^{2} \tau_{\max },
$$

where the coefficient $k_{2}$ depends on the ratio of width to thickness $w / t$ and is about $1 / 3$ for $w / t>10$. Let us assume that the MT starts when $\tau_{\max }$ becomes $\tau_{M}$. The start of the MT is determined to be at the point where the calculated value of the torque coincides with the value obtained from the torsion test, as shown by the solid circle at point $S_{M}$ in Fig. 2(a). As it can be seen, the rate of increase in the torque gradually rises after the MT starting point.

Energy storage and dissipation. The area under the loading curve of the relation between the torque and the angle of twist corresponds to the work done during loading ${ }^{[7]}$. The area under the unloading curve corresponds to the recoverable strain energy $E_{r}$. The area inside the hysteresis loop during loading and unloading corresponds to the dissipated work $W_{d}$.

The relations between $E_{r}$ and $W_{d}$ and temperature $T$ at $\theta=78.5 \mathrm{rad} / \mathrm{m}$ (total angle of twist $\phi=\pi$ ) for both the as-received and heat-treated materials are shown in Fig. 3. It can be seen that $E_{r}$ increases markedly in proportion to $T$ as the torque during unloading increases with increase of temperature. The relation between $E_{r}$ and $T$ is expressed by the following equation

$$
\begin{aligned}
& E_{r}=b\left(T-A_{s}\right), \\
& b=1.86 \mathrm{~mJ} / \mathrm{K}, A_{s}=295 \mathrm{~K}: \text { As-received, } \\
& b=3.68 \mathrm{~mJ} / \mathrm{K}, A_{s}=320 \mathrm{~K}: \text { Heat-treated, }
\end{aligned}
$$

where $A_{s}$ denotes the reverse transformation starting temperature under no stress. The calculated results represented by the solid lines in Fig. 3 agree well with the experimental ones. Therefore the characteristics of energy storage in a torsion bar are superior at high temperatures in the superelastic region. The relation between $E_{r}$ and $T$ reflects the fact that the reverse transformation stress, which

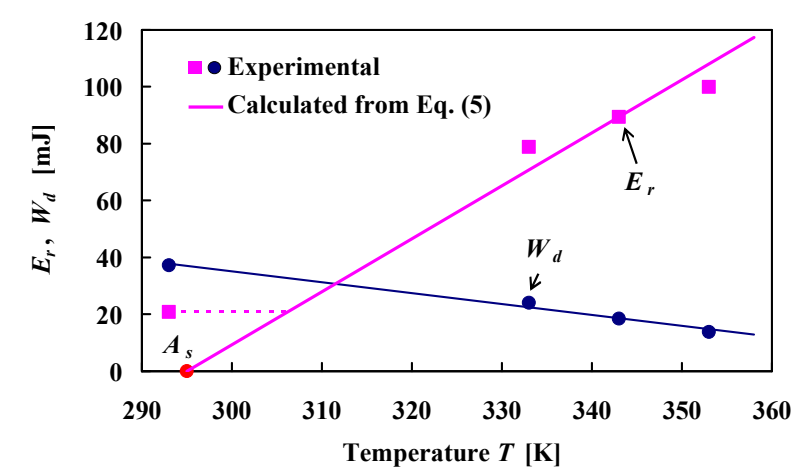

(a) As-received material.

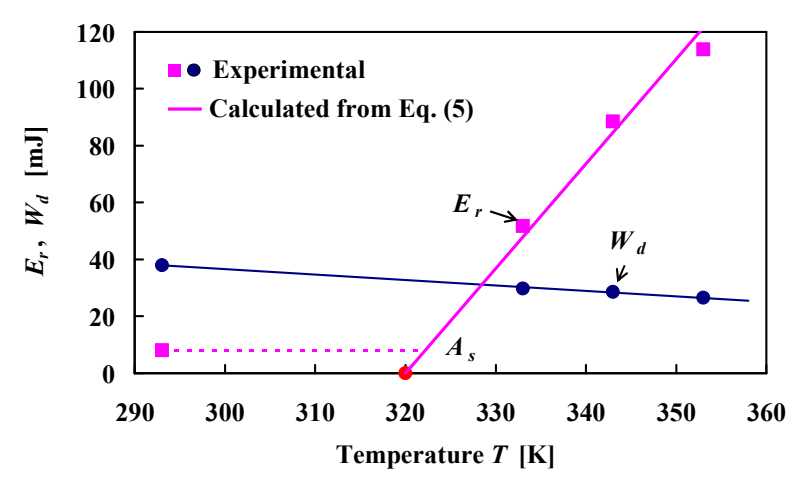

(b) Heat-treated material.

Fig. 3 Dependence of $E_{r}$ and $W_{d}$ on temperature at $\theta=78.5 \mathrm{rad} / \mathrm{m}(\phi=\pi)$. 
acts directly upon $E_{r}$, increases proportionally to temperature $\left(T-A_{s}\right) . E_{r}$ increases with an increase of $\theta$. The precise nature of this dependence of $E_{r}$ on $\theta$ is a question for future enquiry.

The dissipated work $W_{d}$ decreases gradually with an increase of temperature $T$ (Fig. 3). The rate of $W_{d}$ decrease is small and $W_{d}$ is only slightly dependent on $T$. The reason why $W_{d}$ decreases at a high temperature is because the torques at both the MT starting point and the reverse-transformation finishing point increase with rising temperature. Therefore, the torque range for the $M$ - $\phi$ hysteresis loop decreases during loading and unloading. The dependence of $E_{r}$ and $W_{d}$ on temperature in the thin strip under torsion is similar to that for a TiNi SMA wire under tension ${ }^{[7]}$.

Recovery torque. The relation between recovery torque $M$ and angle of twist $\theta$ for the heat-treated material obtained from the recovery torque test is shown in Fig. 4. In Fig. 4, recovery torque $M$ expresses the torque appearing at each temperature $T$ after heating. Recovery torque $M_{e}$, which is a cyclic thermal effect, is defined as the difference between the torque appearing at each heating temperature and the torque obtained at room temperature for the given angle of twist.

As it can be seen in Fig. 4(a), the recovery torque increases proportionally to the angle of twist per unit length $\theta$. The recovery torque also increases with an increase of temperature for the same twist angle per unit length $\theta$. As seen in Fig. 4(b), effective recovery torque $M_{e}$ increases proportionally to temperature rise $\Delta T$. This relationship is approximated by the following equation

$$
M_{e}=c_{1} \theta\left(T-A_{s}\right),
$$

where $A_{s}$ denotes the reverse transformation starting temperature without stress and $A_{s}=320 \mathrm{~K}$. The relation between $M_{e}$ and $T$ is a result of the fact that the recovery stress which directly acts upon $M_{e}$ increases in the reverse transformation region which is proportional to temperature $\left(T-A_{S}\right)$.

Let us discuss the relationship between effective recovery torque $M_{e}$ and angle of twist per unit length $\theta$ based on the theory of elasticity ${ }^{[5]}$. The relation between torque $M$ and $\theta$ for a rectangular-section bar in torsion is expressed by Eq. (2). Considering the relation between $M$ and $\theta$ expressed by Eq. (2), Eq. (6) becomes

$$
M_{e}=c w t^{3} G \theta\left(T-A_{s}\right),
$$

where the coefficient $c$ depends on the ratio $w / t$. The calculated results where $G=20 \mathrm{GPa}$ and $c=1.06 \times 10^{-2} \mathrm{~K}^{-1}$ are shown by the solid lines in Fig. 4(b). It can be seen that the overall inclination of $M_{e}$ is expressed by Eq. (7). However the calculated results do not precisely match the experimental results. This point requires further investigation.

Rotary movement of opening and closing door model. Photographs of the rotary movement of an opening and closing door model using the SMA thin strip (width $w=5 \mathrm{~mm}$ and thickness $t=0.25 \mathrm{~mm}$ ) under heating are shown in Fig. 5. The rotational angle of the door $\phi=\pi / 2$ given at a low temperature diminishes to $\phi=0$ upon heating.

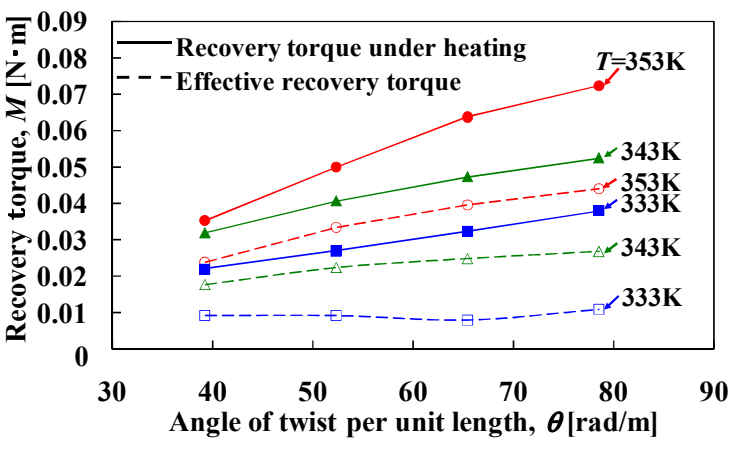

(a) Relation between recovery torque and angle of twist.

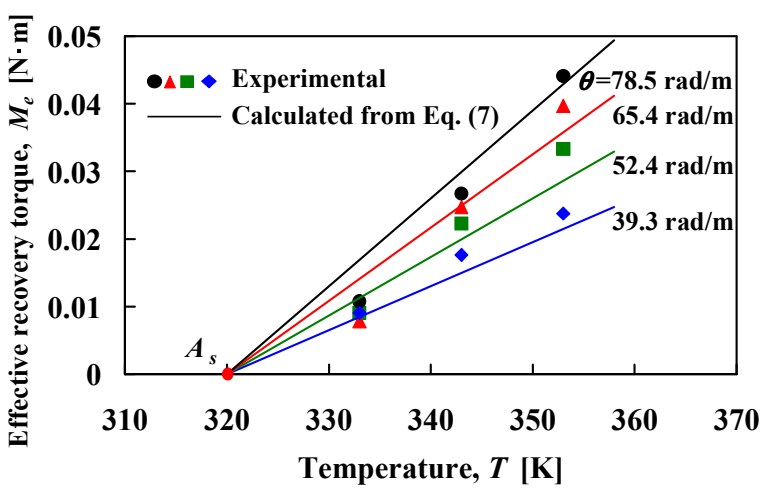

(b) Relation between effective recovery torque and temperature.

Fig. 4 Recovery torque for heat-treated material. 


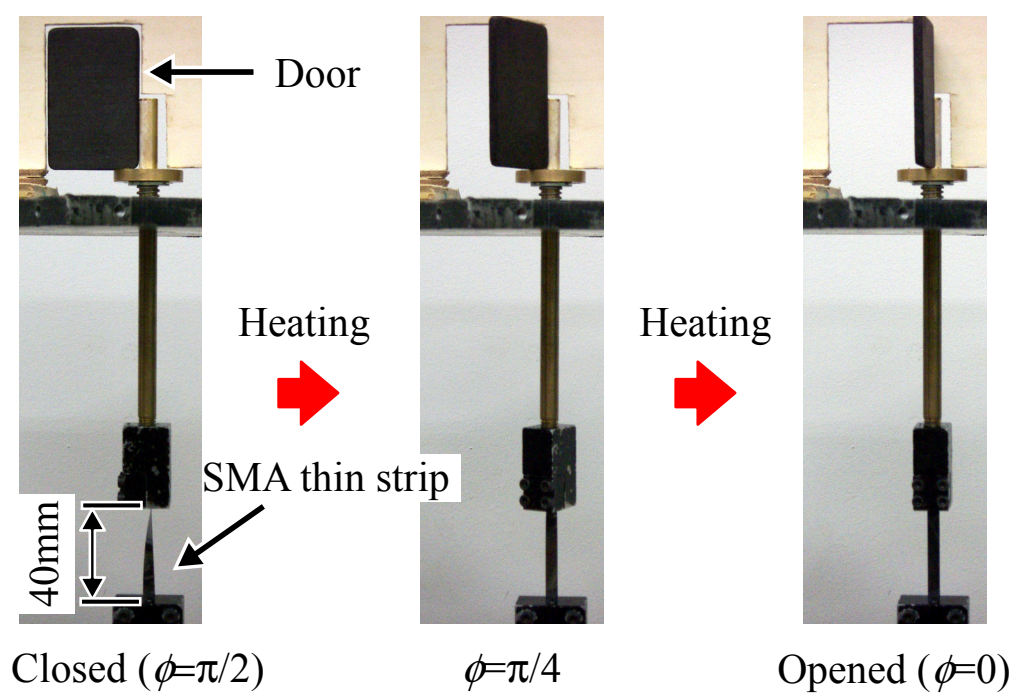

Fig. 5 Rotary movement of a door driven by SMA thin strip under heating.

Thus, if an SMA thin strip is used, a rotary driving element with a small and simple mechanism can be developed. Although this model shows only one-way movement, a two-way element can be developed by using a bias element.

\section{Conclusions}

In order to investigate the rotary driving characteristics of an SMA thin strip, the torsional deformation properties of the strip were discussed. The results obtained can be summarized as follows.

(1) Torque increases in proportion to angle of twist and temperature $\left(T-M_{s}\right)$. Although the recoverable strain energy increases in proportion to temperature $\left(T-A_{S}\right)$, the dissipated work decreases slightly with an increase in temperature.

(2) The recovery torque increases in proportion to angle of twist and temperature $\left(T-A_{s}\right)$.

(3) It is confirmed that a rotary driving element with a small and simple mechanism can be developed by using the TiNi SMA thin strip.

\section{References}

[1] H. Funakubo (ed): Shape Memory Alloys (Gordon and Breach Science Pub., 1987) pp. 1-60.

[2] K. Otsuka and C. M. Wayman (eds): Shape Memory Materials (Cambridge University Press, 1998), pp. 1-49.

[3] D. C. Lagoudas (ed): Shape memory Alloys, Modeling and Engineering Applications (Springer, 2008) pp. 1-52.

[4] J. H. Mabe, R. T. Ruggeri, E. Rosenzweig and C. J. Yu: Smart Struct. Mater : 2004, Proc. SPIE. 5388 (2004), pp. 95-109.

[5] S. T. Timoshenko and J. N. Goodier: Theory of Elasticity, 3rd ed. (McGraw-Hill, 1982), pp. 307-313.

[6] K. Hashimoto, M. Tokuda, T. Inaba, A. Shiode, P. Sittner and B. Bundara: Key Engineering Materials. Vols. 233-236 (2003), pp. 547-552.

[7] E. Pieczyska, S. Gadaj, W. K. Nowacki, K. Hoshio, Y. Makino and H. Tobushi: Science and Technology of Advanced Materials. 6 (2005), pp. 889-894. 